\title{
Dielectrically confined excitons and polaritons in natural superlattices - perovskite lead iodide semiconductors
}

\author{
N.A. GIPPIUS, T. ISHIHARA* , L.V. KELDYSH ${ }^{* *}$, E.A. MULJAROV and S.G. TIKHODEEV \\ General Physics Institute, 38 Vavilova str., Moscow 117333, Russia \\ * Dept. of Physics, Faculty of Sci., Tohoku University, Sendai 980, Japan \\ ${ }^{* *}$ P.N. Lebedev Physical Institute, Moscow, Russia
}

\begin{abstract}
We present the results of calculations of excitonic binding energies, wave functions, diamagnetic factors, and polariton reflection spectra for perovskite lead iodide compounds - naturally grown semiconductor/insulator multi-quantum-well superlattices, with taking into account the interaction of electrons and holes with self-image charges, the image-potential-mediated Coulomb interaction, and the inhomogeneity of electricfield configuration.
\end{abstract}

Currently there is a great deal of interest in naturally grown superlattices - lead iodide based layered semiconductors $\left(\mathrm{C}_{n} \mathrm{H}_{2 n+1} \mathrm{NH}_{3}\right)_{2} \mathrm{PbI}_{4}, n=4,6,8,9,10,12$ [abbreviated as $\mathrm{C}_{n}-\mathrm{PbI}_{4}$ ] and $\left(\mathrm{C}_{6} \mathrm{H}_{5}-\mathrm{C}_{2} \mathrm{H}_{4} \mathrm{NH}_{3}\right)_{2}\left(\mathrm{CH}_{3} \mathrm{NH}_{3}\right)_{m-1} \mathrm{~Pb}_{m} \mathrm{I}_{3 m+1}, m=1,2$ [abbreviated as $\mathrm{PhE}_{-\mathrm{PbI}_{4}}(m=1)$ and $\left.\mathrm{PhE}-\mathrm{Pb}_{2} \mathrm{I}_{7}(m=2)\right]^{1-5}$. This interest is accounted for by the strong excitonic effects in these substances, which make them promising new materials for optoelectronics. Exciton binding energies as large as $E_{e x}=320 \mathrm{meV}$ and pronounced polarization-dependent excitonic polariton effects were reported ${ }^{3}$.

Lead iodide perovskite compounds may be regarded ${ }^{2}$ as natural semiconductor/insulator multi-quantum-well (S/I MQW) superlattices (SLs) consisting of semiconductor lead iodide layers sandwitched between insulator barrier alkylammonium layers (whose gap is twice as large as that of the lead iodide layers). It was shown in ${ }^{2-4}$ that the exciton enhancement in these natural S/I MQWs may be attributed to alternating semiconductor and insulator layers with considerably different dielectric constants, causing the image-potential-mediated modification of electron-hole (e-h) interaction and so-called dielectric confinement of excitons ${ }^{6}$.

In the present paper we calculate the exciton parameters in lead iodide compounds taking into account their SL structure and the image potential including the self-image corrections (arising due to the interaction of electrons and holes with their own image charges). Describing the superlattice structure of the compounds, we follow the Guseinov's approach ${ }^{7}$. As to the selfimage terms, we include them into the $\mathrm{QW}$ localizing potential, thus renormalizing the $\mathrm{QWs}$ states.

Our calculation evolves two succesive steps: ( $i$ ) we find the MQW one-electron and onehole wavefunctions of perpendicular motion with accounting for the interaction with self-image charges, and (ii) solve Schrödinger equation for in-plain excitonic wave-function with averaged (over MQW wave-functions) image-potential-mediated Coulomb interaction.

We assume the excitonic state is of a Wannier-Mott type. This assumption can be selfconsistently verified: the calculated mean e-h distance in an exciton $a_{\mathrm{ex}}$ is large compared to the interatomic distance $a_{0}$ (about 12 and 3-6 $\AA$, respectively). However, we have to ascertain 
that the Wannier-Mott condition $a_{\mathrm{ex}} \gg a_{0}$ is on the verge of its validity. In our calculations we are using envelope-function approximation (EFA) conventional for SLs and MQWs ${ }^{9}$. The only distinction from the known theory is allowing for the image potentials. Our point when choosing the material parameters of $\mathrm{S} / \mathrm{I}$ layers is to use that of $\mathrm{PbI}_{2}$ (for semiconductor layers) and of structurally close organic material (for insulator layers), as it was done in ${ }^{4}$. The detailed description of our calculation procedure see in Ref. ${ }^{10}$.

Table 1 shows certain known material parameters of lead iodide compounds, of $\mathrm{PbI}_{2}$ and organic materials $\mathrm{C}_{n} \mathrm{H}_{2 n+1} \mathrm{NH}_{2}$ and $\mathrm{C}_{6} \mathrm{H}_{5}-\mathrm{C}_{2} \mathrm{H}_{4} \mathrm{NH}_{2}$, along with the results of our calculations of excitonic binding energies $E_{\mathrm{ex}}$, radii $a_{\mathrm{ex}}$, and diamagnetic factors $c_{0}$.

We have chosen $\mathrm{C}_{10}-\mathrm{PbI}_{4}$ as a starting point in our calculations and fitted our theory to the experimental values of $E_{\text {ex }}$ and $c_{0}$, shown in Tab. 1. When fitting, we have mostly used two adjustable parameters: the ionic radius of iodide atoms $R_{I}$, which we added (following ${ }^{3,4}$ ) to the geometric width of the $\mathrm{PbI}_{4}$ layer $l_{w}^{0}$ (shown in Tab. 1), $l_{w}=l_{w}^{0}+2 R_{I}, \quad l_{b}=l_{b}^{0}-2 R_{I}$ and reduced excitonic mass $\mu$. We have also assumed the dielectric constant of well layers $\varepsilon_{w}=6.1$ (see Tab. 1) and chosen that of barrier layers $\varepsilon_{b}$ from the condition $\varepsilon_{\infty}=\left[\varepsilon_{w} l_{w}+\varepsilon_{b} l_{b}\right] /\left[l_{w}+l_{b}\right]$. Thus we have guaranteed the correct experimental value of high frequency dielectric constant $\varepsilon_{\infty}$. As a rule, calculated in such a way $\varepsilon_{b}$ is close to that shown in Tab. 1 for $\mathrm{C}_{n} \mathrm{H}_{2 n+1} \mathrm{NH}_{2}$.

We have obtained better agreement with the experimental values of $E_{\text {ex }}$ and $c_{0}$ choosing $\mu=0.21 m_{0}$ and $R_{I}=1 \AA$. After fitting our theory to the experimental data on $\mathrm{C}_{10}-\mathrm{PbI}_{4}$, we have calculated the excitonic parameters in other lead iodide compounds, using the same $\mu=0.21 \mathrm{~m}_{0}$ and $R_{I}=1 \AA, \varepsilon_{w}=6.1$ and changing accordingly to Tab. $1 l_{w, b}^{0}, \varepsilon_{\infty}$ (when the latter is known). The results of our calculations of $E_{\text {ex }}, c_{0}$ and $a_{\text {ex }}$ are shown in Tab. 1. One can see that although our fitting procedure was rather crude and the number of unknown material parameters was large, theoretical results show reasonable agreement with the experimental data for the whole lead iodide family. The fact that our theory gives different excitonic parameters for different members of $\mathrm{C}_{n} \mathrm{PbI}_{4}$ family, clearly demonstrates the role of SL structure. (Within our approach the well layers are the same for all $\mathrm{C}_{n} \mathrm{PbI}_{4}$ compounds.) Our accounting for self-image corrections, which is another difference of our approach from the previous one ${ }^{4}$, manifests itself in larger fitted value for reduced excitonic mass $\left(\mu=0.21 m_{0}\right.$ in our calculations and $0.1 m_{0}$ in $\left.{ }^{4}\right)$.

The polarization-resolved optical experiments ${ }^{2,3}$ using EM wave with frequency close to the polariton resonance show a large difference in light reflection for two polarizations of electromagnetic wave $\vec{E} \perp \vec{c}$ and $\vec{E} \| \vec{c}$ (where $\vec{c}$ is a normal to layers of the superlattice). This seeming anysotropy of the exciton oscillator strength may be explained by essentially different spatial configurations of the electric field in the S/I SLs for these two polarizations. If $\vec{E} \perp \vec{c}$, the electric field is uniform, whereas at $\vec{E} \| \vec{c}$ the electric field in well layers (just where the excitons are confined to) is much smaller than in the barrier ones, thus causing a decrease of excitonic oscillator strength. It is also clear, that for $\vec{E} \| \vec{c}$ polarization the exciton-photon interaction has to depend drastically on the exciton wavefunction.

The results of our calculations of the polariton reflection spectra within the nonlocal theory of Ref. ${ }^{11}$ are illustrated in Fig.1. Note a drastic reflectivity dependence on the exciton formfactor for $\vec{E} \| \vec{c}$ polarization. The reflection spectra for $\vec{E} \perp \vec{c}$ polarization does not depend on the exciton formfactor at all. 
Table 1: Experimental and calculated parameters of lead iodide compounds: high-frequency dielectric constant $\varepsilon_{\infty}$, band gap $E_{g}, \mathrm{~S} / \mathrm{I}$ layer width $l_{\mathrm{w}}^{0} / l_{\mathrm{b}}^{0}$, exciton binding energy, diamagnetic factor and mean radii $E_{\text {ex }}, c_{0}$ and $a_{\text {ex }}$

\begin{tabular}{|c|c|c|c|c|c|c|c|c|c|}
\hline \multirow[t]{3}{*}{$\overline{\text { Compound }}$} & \multirow[t]{3}{*}{$\varepsilon_{\infty}$} & \multirow{3}{*}{$\begin{array}{c}E_{g} \\
\\
\mathrm{eV} \\
(\mathrm{T}=1.6 \mathrm{~K})\end{array}$} & \multirow{3}{*}{$\begin{array}{l}\overline{\overline{l_{\mathrm{w}}^{0}}} \\
\AA\end{array}$} & \multirow{3}{*}{$\begin{array}{l}\overline{\overline{l_{b}^{0}}} \\
\AA\end{array}$} & \multicolumn{2}{|l|}{$\overline{\overline{E_{\text {ex }}}}$} & \multicolumn{2}{|c|}{$\bar{c}$} & \multirow{3}{*}{$\begin{array}{c}a_{\mathrm{ex}} \\
\AA \\
\AA \\
\text { theor }\end{array}$} \\
\hline & & & & & \multicolumn{2}{|l|}{$\mathrm{meV}$} & \multicolumn{2}{|c|}{$10^{-7} \frac{\mathrm{eV}}{T^{2}}$} & \\
\hline & & & & & $\exp$ & theor & $\exp$ & theor & \\
\hline $\mathrm{PbI}_{2}$ & $6.1^{\mathrm{c}}$ & $2.53^{b}$ & & & $30^{6}$ & & $9.7^{i}$ & & $40^{6}$ \\
\hline & & & & & $63^{e}$ & & & & $19^{e}$ \\
\hline $\begin{array}{l}\mathrm{C}_{n} \mathrm{H}_{2 n+1}- \\
\mathrm{NH}_{2}\end{array}$ & $\begin{array}{c}2.1^{\mathrm{d}} \\
(n=10)\end{array}$ & $\begin{array}{l}5.5^{\mathrm{g}} \\
(n=12)\end{array}$ & & & & & & & \\
\hline & $2.07^{\mathrm{h}}$ & & & & & & & & \\
\hline $\begin{array}{l}\mathrm{C}_{6} \mathrm{H}_{5}- \\
\mathrm{C}_{2} \mathrm{H}_{4} \mathrm{NH}_{2}\end{array}$ & $2.34^{\mathrm{h}}$ & & & & & & & & \\
\hline $\mathrm{C}_{4}-\mathrm{PbI}_{4}$ & & & $6.36^{k}$ & $\begin{array}{r}8.53^{8} \\
8.81^{1}\end{array}$ & $290 \pm 20^{8}$ & $289^{\dagger}$ & & $1.48^{\dagger}$ & $11.9^{\dagger}$ \\
\hline $\mathrm{C}_{6}-\mathrm{PbI}_{4}$ & & & $6.36^{\mathrm{k}}$ & $10.03^{1}$ & & $285^{\dagger}$ & $\begin{array}{r}2.16- \\
3.53^{\mathrm{a}}\end{array}$ & $1.60^{t}$ & $12.3^{\dagger}$ \\
\hline $\mathrm{C}_{8}-\mathrm{PbI}_{4}$ & & & $6.36^{\mathrm{k}}$ & $12.02^{1}$ & & $288^{\dagger}$ & & $1.70^{\dagger}$ & $12.7^{\dagger}$ \\
\hline $\mathrm{C}_{9}-\mathrm{PbI}_{4}$ & & & $6.36^{k}$ & $13.53^{1}$ & $320 \pm 30^{8}$ & $293^{\dagger}$ & & $1.70^{f}$ & $12.7^{\dagger}$ \\
\hline $\mathrm{C}_{10}-\mathrm{PbI}_{4}$ & $3.24^{3}$ & $2.87^{i}$ & $6.36^{k}$ & $14.89^{I}$ & $320 \pm 30^{8}$ & $301^{t 7}$ & $1.7^{i}$ & $1.68^{f f}$ & $12.7^{f f}$ \\
\hline $\mathrm{C}_{12}-\mathrm{PbI}_{4}$ & $3.39^{k}$ & & $6.36^{\mathrm{k}}$ & $18.15^{\mathrm{k}}$ & $310 \pm 30^{8}$ & $317^{\dagger}$ & & $1.63^{\dagger}$ & $12.5^{\dagger}$ \\
\hline $\mathrm{PhE}-\mathrm{PbI}_{4}$ & $4.41^{\mathrm{k}}$ & $2.58^{k}$ & $\begin{array}{l}6.36^{\mathrm{k}} \\
6.41^{\mathrm{j}}\end{array}$ & $\begin{array}{l}9.82^{k} \\
9.85^{j}\end{array}$ & $220 \pm 30^{k}$ & $269^{f}$ & & $1.60^{\dagger}$ & $12.4^{\dagger}$ \\
\hline $\mathrm{PhE}-\mathrm{Pb}_{2} \mathrm{I}_{7}$ & & $2.30^{k}$ & $\begin{array}{l}12.70^{\mathrm{k}} \\
13.05^{\mathrm{j}}\end{array}$ & $9.71^{j}$ & $170^{k}$ & $223^{\dagger}$ & & $1.95^{\dagger}$ & $13.6^{\dagger}$ \\
\hline
\end{tabular}

${ }^{\mathrm{a} T}$ T. Kataoka, T. Kondo, R. Ito, S. Sasaki, K. Ushida, and S. Miura, Phys. Rev. B, 47, 2010 (1993).

'Le Chi Thanh, et al., J. Phys. Chem. Solids 36, 699 (1975)

${ }^{c}$ G. Lucovsky, et al., Solid State Commun. 18, 811 (1976)

${ }^{d}$ CRC Handbook of Chemistry and Physics, 63rd ed., Chem. Rubber Comp., Boca Raton, FL, 1983

'Y. Nagamune, S. Takeyama, and N. Miura, Rhys. Rev. B 40, 8099 (1989)

${ }^{\mathrm{f}}$ Ref. 2

8Ref. 3

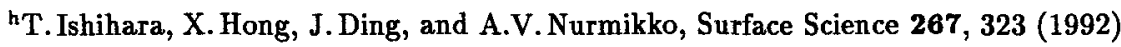

iRef. 11

jJ. Calabrese et al., J. Am. Chem. Soc. 113, 2328 (1991)

${ }^{k}$ Ref. 4

'T. Ishihara, unpublished

$\dagger$ Theor, this work

${ }^{\dagger \dagger}$ Fitted number, this work 


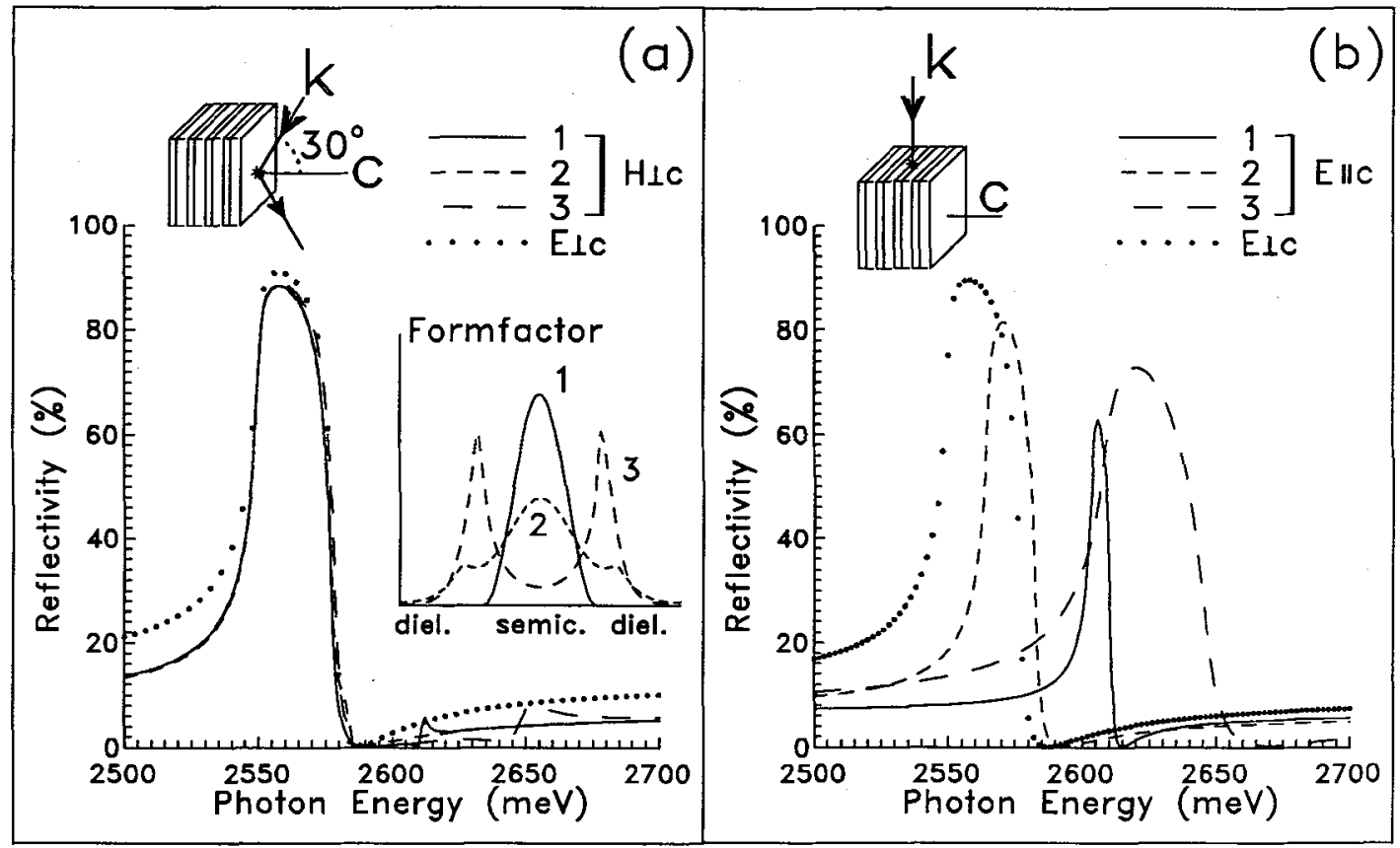

Fig.1 The reflection spectra of the S/I SL for $30^{\circ}$ angle of incidence (a) and beam directed along the SL layers (b) calculated for three different exciton formfactors $\psi_{\text {exc }}\left(z_{e}=z_{h}=z, \rho=0\right)$, shown in the inset.

\section{REFERENCES}

1 S.S. Nagapetyan, Yu.I.Dolzhenko, E.R. Arakelova, V.Koshkin, Yu.T.Struchkov, and V.E. Shklover, Zh. Neorg. Khim., vol. 33, pp. 2806-2812, 1988 [Russ. J. Inorg. Chem., vol. 33, pp. 1614, 1988].

${ }^{2}$ T. Ishihara, J. Takahashi, and T. Goto, Solid State Commun., vol. 69, pp. 933-936, 1989.

3 T. Ishihara, J. Takahashi, and T. Goto, Phys. Rev. B vol. 42, pp. 11099-11107, 1990.

4 X. Hong, T. Ishihara, and A.V. Nurmikko, Phys. Rev. B, vol. 45, pp. 6961-6965, 1992.

${ }^{5}$ J. Calabrese, N.L. Jones, R.L. Harlow, N. Herron, D.L. Thorn, and Y. Wang, J. Am. Chem. Soc. vol. 113, pp. 2328-2330, 1991.

${ }^{6}$ L.V. Keldysh, Pis'ma. Zh. Eksp. Teor. Fiz., vol. 29, pp. 716-719, 1979 [JETP Lett., vol. 29, p. 658,1979$]$.

${ }^{7}$ R.R. Guseinov, Phys. Stat. Solidi (b), vol. 125, pp. 237-243, 1984.

${ }^{8}$ C. Xu, H. Sakakura, T. Kondo, S. Takeyama, N. Miura, Y. Takahashi, K. Kumata, and R. Ito, Solid State Commun., vol. 79, pp. 249-253, 1991.

${ }^{9} \mathrm{G}$. Bastard, Wave Mechanics Applied to Semiconductor Heterostructures, Les Editions de Physique, Les Ulis, France, 1988.

${ }^{10}$ E.A. Muljarov, S.G. Tikhodeev, and T. Ishihara, Optical Engineering, vol. 1985, 1993.

${ }^{11}$ L.V. Keldysh, Superlattices \& Microstructures, vol. 4, pp. 637-642, 1988. 